\title{
OPEM
}

www.opem.org

Oriental Pharmacy and Experimental Medicine 2008 8(2), 178-186

DOI 10.3742/OPEM.2008.8.2.178

\section{Preparation and characterization of ibuprofen-loaded alginate microspheres using ethylenediamine as a crosslinker}

\author{
Sabyasachi Maiti ${ }^{1 * *}$ and Biswanath $\mathrm{Sa}^{2}$ \\ ${ }^{1}$ Gupta College of Technological Sciences, Ashram More, GT Road, Asansol, India; ${ }^{2}$ Department of Pharmaceutical \\ Technology, Jadavpur University, Kolkata, India
}

Received for publication May 09, 2007; accepted October 16, 2007

\begin{abstract}
SUMMARY
In this study, ionotropic gelation method was used for the preparation of ibuprofen-loaded calcium alginate (CALG) and ethylenediamine (EDA) treated calcium alginate (EDA-CALG) microspheres. The effect of EDA-treatment on drug entrapment efficiency, particle size, morphology, swelling behavior and in vitro release characteristics of the microspheres was investigated by varying its concentration from 0.5 to $2 \%(\mathrm{v} / \mathrm{v})$. The reduction in drug entrapment efficiency by a maximum of $44.60 \%$ was noted for EDA-CALG microspheres compared to untreated CALG microspheres. The particle size and swelling index of EDA-CALG microspheres were reduced with increasing EDA concentration. All the microspheres were observed to retain their spherical shapes with rough surfaces. EDA-CALG microspheres prepared using $1 \%$ and $2 \% \mathrm{v} / \mathrm{v}$ EDA, released almost all of its content within $7 \mathrm{~h}$ in $\mathrm{pH} 6.8$ phosphate buffer, however, CALG microspheres were found to release the same within $3 \mathrm{~h}$. The intensity of melting endothermic peak of ibuprofen reduced significantly at lower drug load as experienced from DSC thermograms. The FT-IR spectrum of pure ibuprofen, ibuprofen-loaded CALG and EDA-CALG microspheres showed the characteristic band of $\mathrm{C}=\mathrm{O}$ stretching vibration of ibuprofen. Hence, this study revealed that EDA can be employed for the preparation of ibuprofen-loaded CALG microspheres to retard the drug release to some extent.
\end{abstract}

Key words: Ibuprofen; Alginate; Ethylenediamine; Microspheres; Ionotropic gelation

\section{INTRODUCTION}

Many microencapsulation techniques have been developed to mask the unpleasant taste of drugs, to improve the stability and control the release of drugs from delivery system (Bodmeier and Wang, 1993; Calis et al., 2002). Currently, much attention

*Correspondence: Sabyasachi Maiti, Department of Pharmaceutics, Gupta College of Technological Sciences, Ashram More, G.T. Road, Asansol - 713301, West Bengal, India. Tel: +919474119931; Fax: +913412214602; E-mail: sabya24@yahoo.co.in has been paid to the formulation of multiunit microparticulate dosage forms (Tamilvanan and Sa, 1999) because they avoid the vagaries of gastric emptying and different transit rates through the gastro intestinal tract, thereby, release drugs more uniformly (Follonier and Doelkar, 1992). Moreover, a multiunit system spreads over a large area of the absorbing mucosa and prevents exposure to a high drug concentration, when compared to single unit dosage form on chronic dosing (Davis et al., 1984). This fact has given impetus to the development of oral microparticulate systems for drug delivery. 
Recently, the techniques such as spray drying, phase separation-coacervation, emulsification-solvent evaporation are most widely being used for the preparation of microparticles (Sinha and Trehan, 2003). However, a large volume of organic solvents is required for their processing and the problems of toxicity, the possibility of explosion and air pollution associated with their use has paid much attention to the development of microparticles in a completely aqueous environment (Sa et al., 1991). Moreover, the synthetic nonbiodegradable polymers used for their preparation pose the problems of toxicity. Thus, biodegradable natural polymers are being investigated for the preparation of microparticulate dosage forms because of their biocompatibility, biodegradation and non-toxicity on administration (Takka and Acarturk, 1999).

Alginates are naturally occurring polysaccharide obtained mainly from marine brown algae belonging to phaeophyceae, composed of two monomeric units $\alpha$-L-guluronic acid and $\beta$-D-mannuronic acid. Among the polysaccharides, sodium alginate has been studied extensively as an encapsulating material for controlled drug delivery (Gursoy and Cevik, 2000). Although, alginate microparticles are stable in acidic media but easily swell and disintegrate in alkaline media (Acarturk and Takka, 1999). As calcium ions are being released by the ion exchange with sodium ions of the alkaline medium, electrostatic repulsion between the carboxylate anions further accelerate the swelling and erosion of alginate gels (Kikuchi et al., 1997) and hence calcium alginate matrix alone do not seem suitable as an oral controlled release system (Ostberg et al., 1994). In order to improve the stability of alginate gels, complex coacervation of oppositely charged costlier polyelectrolyte such as chiotsan (Coppi et al., 2001; Anal et al., 2003; Bhopatkar et al., 2005), poly-l-lysine (Rajaonarivony et al., 1993; Thu et al., 1996; Robinson and De, 2003) and polyethyleneimine (Haldar et al., 2005) has been attempted. Ethylenediamine (EDA) is an analogue of the dialcohol ethylene glycol. It is commonly used as textile lubricant, fungicides, insecticides, corrosion inhibitor in antifreeze and in the paint industry. It also finds its application in some medicaments as a chemical stabilizer and in the manufacture of other drugs such as aminophylline and some antihistamines. However, the use of ethylenediamine as a crosslinking agent in the preparation of drug-loaded calcium alginate (CALG) microspheres is in its infancy. It has been reported that a new alginate matrix can be produced using ethylenediamine to covalently crosslink the alginate carboxyl groups to stabilize and control the release of growth factors (Tanihara et al., 2001). Another report indicated that propylene glycol alginate may be covalently crosslinked with ethylenediamine under basic conditions (Ronan and Thompson, 2000). Recently alginic acid was modified by carbodiimide-mediated linkage of several heterocyclic and aromatic amines to the carboxyl groups of alginate giving the alginate polycation characteristics through which binding to the nucleic acids could be facilitated (Padmanabhan and Smith, 2002). In human populations, no reports have been found about genotoxicity, carcinogenicity, or reproductive toxicity following exposure to EDA (Cary et al., 1999). In a three month study when Fisher 344 rats were given 0.05 to $1 \mathrm{~g}$ EDA hydrochloride/ $\mathrm{kg}$ BW/ day $\left(\mathrm{LD}_{50} 3.25 \mathrm{~g} / \mathrm{kg}\right)$, no increase in mortality was observed even at the highest dose level (Yang et al., 1983; Sheftel, 1995). Ibuprofen is an oral nonsteroidal anti-inflammatory drug and is the first phenylalkamine acid approved by FDA for general analgesic use. Because of its shorter biological half-life, hazards of gastric irritation and bronchocontriction; it is an appropriate candidate for microencapsulation (Dubernet et al., 1990; Weib et al., 1993) and controlled drug delivery. Therefore, the objective of our present study was to prepare ibuprofen-loaded CALG microspheres using EDA and investigate the effect of EDA on drug entrapment efficiency, particle size, surface topography and in vitro release behavior of CALG microspheres. 


\section{MATERIALS AND METHODS}

\section{Materials}

Ibuprofen was a gift from Albert-David Ltd., Kolkata, India. Sodium alginate was supplied by S.D fine-chem Ltd, Mumbai, India. The $0.1 \%$ (w/v) aqueous solution of sodium alginate exhibits a viscosity of $1.15 \mathrm{cp}$ at $25^{\circ} \mathrm{C}$. Ethylenediamine for synthesis (99\%) was purchased from Merck Ltd., Mumbai, India. Calcium chloride dihydrate (98\%) was obtained commercially from Qualigens fine chemicals, Mumbai, India. All other chemicals were of analytical grade and used without further purification.

\section{Preparation of microspheres}

The CALG and EDA-CALG microspheres were prepared following the protocol as described in Table 1 . Sodium alginate $(2 \% \mathrm{w} / \mathrm{v})$ was dissolved in $10 \mathrm{ml}$ of deionized water and ibuprofen (50\% $\mathrm{w} / \mathrm{w}$ of sodium alginate) was uniformly dispersed in the alginate solution. The bubble-free sodium alginate solution was extruded through a 16 gauge flat-tipped needle into $100 \mathrm{ml}$ aqueous solution of calcium chloride. Following gelation for $15 \mathrm{~min}$ in calcium chloride solution, the resultant CALG microspheres were filtered, washed with deionized water $(3 \times 50 \mathrm{ml})$ and dried in vacuum oven until three consecutive weights obtained were constant. The same procedure was adopted for the preparation of EDA-CALG microspheres; the only difference being the composition of the curing solution. The EDA-CALG microspheres were cured in $100 \mathrm{ml}$ aqueous solution of calcium chloride containing different concentration of EDA: 0.5, 1.0 and 2\% v/v.

\section{Entrapment efficiency}

Accurately weighed, $20 \mathrm{mg}$ of dried microspheres were crushed with the help of a mortar and pestle and were transferred into a $250 \mathrm{ml}$ volumetric flask. About $150 \mathrm{ml}$ of phosphate buffer ( $\mathrm{pH}$ 7.4) USP was added to it and was placed on a mechanical shaker overnight to effect complete drug release into solution. Finally, the volume was adjusted to $250 \mathrm{ml}$ with fresh buffer solution and filtered. Nine milliliters of the filtrate was diluted to $50 \mathrm{ml}$ with fresh pH 7.4 phosphate buffer solution. The diluted samples were analyzed spectrophotometrically at $\lambda_{\max } 221 \mathrm{~nm}$. Each formulation was analyzed in triplicate $(n=3)$. Entrapment efficiency was calculated as follows: Entrapment efficiency $(\%)=($ actual drug loading/theoretical drug loading) $\times 100$.

\section{Particle size analysis}

The size of dried CALG and EDA-CALG microspheres were measured using an optical microscope (Olympus, Model HB and India). A standard stage micrometer was used to calibrate the optical micrometer. The diameters were measured using 50 particles of each formulation and average diameter was computed.

\section{Scanning electron microscopy}

Dried CALG microspheres and EDA-CALG microspheres were mounted onto stubs, using double sided adhesive tape and vacuum coated with gold film using sputter coater (Edward S-150, UK). The coated microspheres were observed under scanning electron microscope (Jeol, ISM5,200 , Japan) for surface characteristics.

\section{Swelling study}

About $20 \mathrm{mg}$ of dried samples of blank CALG and EDA-CALG microspheres were allowed to swell in $40 \mathrm{ml}$ of $0.1 \mathrm{~N} \mathrm{HCl}$ solutions for $2 \mathrm{~h}$ and in phosphate buffer $\mathrm{pH} 6.8$ for $4 \mathrm{~h}$ separately. Samples were withdrawn at different time intervals; surface moisture was removed with tissue paper and weighed. The swelling index was calculated by subtracting initial weight of sample microspheres $\left(W_{\mathrm{o}}\right)$ from weight of microspheres after swelling $\left(W_{s}\right)$ and then divided by the initial weight of sample microspheres $\left(\mathrm{W}_{\mathrm{o}}\right)$.

\section{In vitro release study}

In vitro release of ibuprofen from CALG and EDA- 
CALG microspheres was performed using USP dissolution rate test apparatus, paddle type (Campbell Electronics, Mumbai, India) under sink condition. Accurately weighed, $20 \mathrm{mg}$ of dried microparticles was placed in $900 \mathrm{ml} \mathrm{pH} 6.8$ phosphate buffer USP solution and maintained at $37 \pm 1^{\circ} \mathrm{C}$. The paddle was rotated at $100 \mathrm{rpm}$. Ten milliliters of aliquot was withdrawn from dissolution media at specified time intervals and same volume of fresh medium was replenished immediately. The samples were analyzed spectrophotometrically at the wavelength of maximum absorbance, without further dilution. The in vitro release study in $\mathrm{HCl}$ solution ( $\mathrm{pH}$ 1.2) was carried out under the same experimental condition for $2 \mathrm{~h}$. Cumulative percentage of ibuprofen released in the alkaline media was plotted as a function of time.

\section{Release kinetics}

The mean release data were fitted into KorsmeyerPeppas equation to evaluate the drug release kinetics (Korsmeyer et al., 1983).

$M_{\mathrm{t}} / M_{\alpha}=k t^{n}$

Where $M_{t} / M_{\alpha}$ is the fraction of drug released at time $t ; k$ is a release rate constant comprising the structural and geometric characteristics of the microparticle; and $n$, the release exponent, is a parameter that depends on the release mechanism (Peppas, 1985). Peppas used this $n$ value in order to characterize different release mechanisms. If the $n$ value is 0.5 or less, the release mechanism follows Fickian diffusion, and higher values $0.5<n<1$ indicate a non-Fickian model (anomalous transport). For the determination of the values of $n$ and $k$, the portion of the release curve where $M_{\mathrm{t}} / M_{\infty}<0.6$ should only be used. The values of $n$ and $k$ were obtained from the slope and intercept of a plot of $\log M_{\mathrm{t}} / M_{\infty}$ versus $\log t$, respectively.

\section{Fourier transform infrared spectroscopy (FT-IR)}

FT-IR spectra for completely dried pure ibuprofen, CALG and EDA-CALG microspheres were recorded in a Fourier Transform Infrared spectrophotometer (FT-IR, 410 JASCO, Japan) at a scan speed of $2 \mathrm{~mm} / \mathrm{s}$ from $4,000 \mathrm{~cm}^{-1}$ to $600 \mathrm{~cm}^{-1}$ using $\mathrm{KBr}$ pellets. The accumulations for pure ibuprofen, ibuprofen-loaded CALG and EDA-CALG microspheres were 63, 37 and 42 , respectively.

\section{Differential scanning calorimetry (DSC)}

DSC thermograms of pure ibuprofen, CALG and EDA-CALG microspheres were performed in an atmosphere of nitrogen. Weighed amount of sample was kept in hermetically sealed aluminium pans and heated at a scanning rate of $10^{\circ} \mathrm{C} /$ min over a temperature range chosen on the basis of drug melting point $\left(30-140^{\circ} \mathrm{C}\right)$ using Perkin-Elmer instrument (Pyris Diamond TG/DTA, Singapore). Calibration was performed by using an indium sample.

\section{RESULTS AND DISCUSSION}

In order to investigate the effect of EDA on various physicochemical properties, ibuprofen-loaded CALG and EDA-CALG microspheres were prepared using the droplet extrusion method combined with ionotropic gel formation in presence of either calcium chloride solution or calcium chloride solution containing different concentration of EDA. It is well established that as soon as the droplets of sodium alginate solution contacted with calcium chloride solution, spherical particles were instantaneously formed, due to the rapid establishment of calcium-mediated associations between poly guluronic acid sequences on the polymer backbone (Gombotz and Wee, 1998). Attempts were also made to increase the strength of gel network structure of calcium alginate beads through interaction with polyethyleneimine (Haldar et al., 2005). It has been reported that polyethyleneimine, a positively charged polyelectrolyte can interact with alginate to form a polyelectrolyte complex membrane (ElGibaly and Anwar, 1998). On the other hand, a recent report indicated that EDA can be used to 

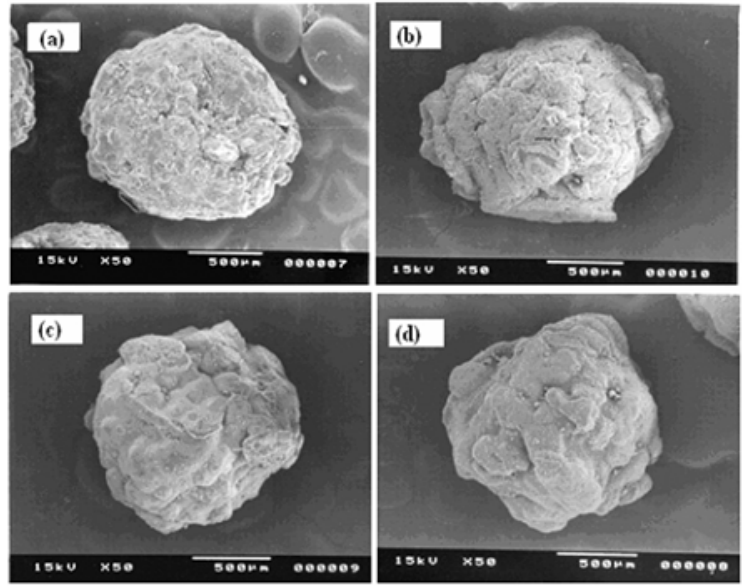

Fig. 1. Scanning electron micrographs for microspheres (a) CALG, (b) 0.5\% EDA-CALG, (c) 1\% EDA-CALG, (d) $2 \%$ EDA-CALG.

covalently crosslink the alginate carboxyl groups to develop a new alginate gel matrix (Tanihara et al., 2001). Therefore, the interaction between protonated amino groups $\left(-\mathrm{NH}_{3}{ }^{+}\right)$of EDA with negatively charged carboxylic acid groups ( $\left.\mathrm{COO}^{-}\right)$of sodium alginate can be utilized to impart a tight gel network structure in the CALG microspheres. Scanning electron micrographs of ibuprofen-loaded CALG and EDA-CALG microspheres has been shown in Fig. 1. It was observed that although, the microspheres retained its sphericity after drying but were not quite smooth in surface. Since ibuprofen is insoluble in polymer solution, pressure difference between the dispersed phase and the external aqueous phase can be expected insignificant and insoluble drug particles may provide a rigid matrix to the microspheres to retain its sphericity (Sa, 1996). A similar type of surface morphology has been observed previously for ibuprofen-loaded CALG beads (Arica et al., 2005). The surface of EDAtreated microspheres appeared to be striated. The striations developed at the surface may be due to the crosslinking reaction between EDA and alginate. The size of the EDA-CALG microspheres was found to decrease with increasing EDA concentration, even when compared to CALG microspheres. The higher contraction of the EDA-CALG microspheres

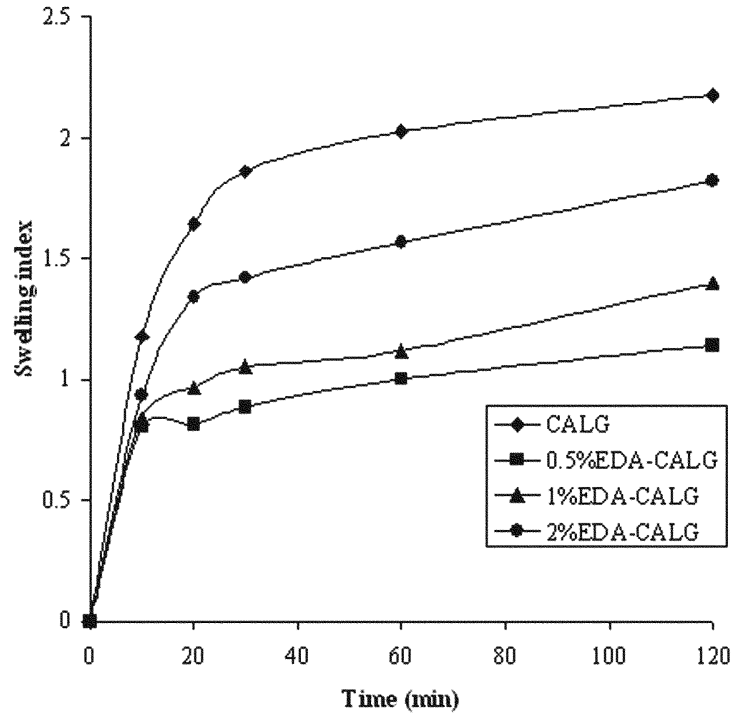

Fig. 2. Swelling study pf blank CALG and EDACALG microspheres in $\mathrm{HCl}$ solution ( $\mathrm{pH}$ 1.2).

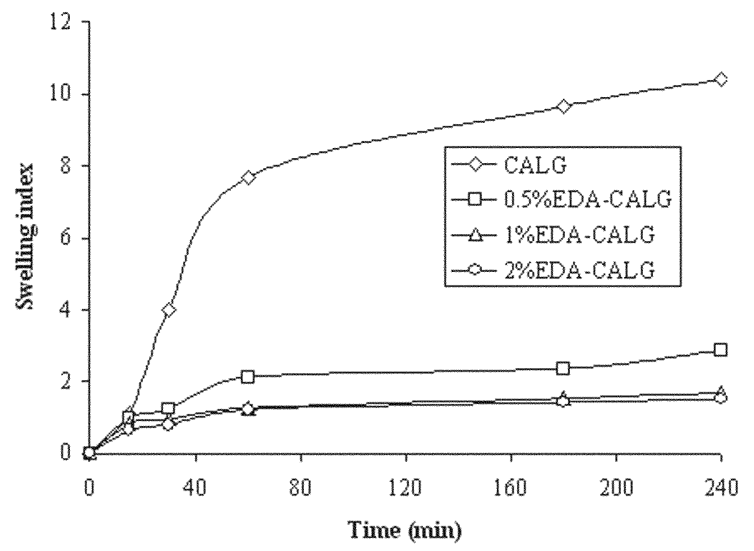

Fig. 3. Swelling study pf blank CALG and EDACALG microspheres in phosphate buffer ( $\mathrm{pH}$ 6.8).

with increasing EDA concentration may be attributed to such differences in mean particle size (Table 2). The swelling behavior of CALG and EDA-CALG microspheres was studied in both $\mathrm{HCl}(\mathrm{pH}$ 1.2) and phosphate buffer ( $\mathrm{pH}$ 6.8) solution and has been represented in Fig. 2 and Fig. 3, respectively. Microspheres with an extra EDA treatment showed lower swelling in both the media. The variation in the concentrations of EDA had a significant effect on the entrapment of ibuprofen in EDA-CALG microspheres. In presence of EDA, the entrapment 
Table 1. Preparative conditions for ibuprofen-loaded ALG and EDA-CALG microspheres with their entrapment efficiency and \% yield

\begin{tabular}{cccccccc}
\hline $\begin{array}{c}\text { Sodium } \\
\text { alginate } \\
(\% \mathrm{w} / \mathrm{v})\end{array}$ & $\begin{array}{c}\text { Calcium } \\
\text { chloride } \\
(\% \mathrm{w} / \mathrm{v})\end{array}$ & $\begin{array}{c}\text { EDA } \\
\text { conc. } \\
(\% \mathrm{v} / \mathrm{v})\end{array}$ & $\begin{array}{c}\text { Curing } \\
\text { time } \\
(\mathrm{min})\end{array}$ & $\begin{array}{c}\text { Drug/algi- } \\
\text { nate ratio }\end{array}$ & $\begin{array}{c}\text { Actual drug } \\
\text { loading } \\
(\% \mathrm{w} / \mathrm{w})\end{array}$ & $\begin{array}{c}\text { Mean drug entrap- } \\
\text { ment efficiency }(\%) \\
\pm \text { S.D. }\end{array}$ & $\begin{array}{c}\% \\
\text { yield* }\end{array}$ \\
\hline 2.0 & 3 & 0.0 & 15 & $1: 1$ & $49.45 \pm 0.76$ & $98.90 \pm 1.52$ & 91.95 \\
2.0 & 3 & 0.5 & 15 & $1: 1$ & $31.76 \pm 1.43$ & $63.52 \pm 2.88$ & 74.26 \\
2.0 & 3 & 1.0 & 15 & $1: 1$ & $27.91 \pm 0.61$ & $55.83 \pm 1.22$ & 70.41 \\
2.0 & 3 & 2.0 & 15 & $1: 1$ & $27.14 \pm 0.51$ & $54.29 \pm 1.01$ & 69.64 \\
\hline
\end{tabular}

"The percentage yield value is defined as the quantity of microspheres produced as a function of loaded drug and polymer.

Table 2. Effect of EDA on particle size and release behavior of ibuprofen in both alkaline (pH 6.8) and acidic ( $\mathrm{pH}$ 1.2) media from CALG microspheres prepared with $2 \%$ sodium alginate

\begin{tabular}{ccccccc}
\hline $\begin{array}{c}\text { Microsphere } \\
\text { type }\end{array}$ & $\begin{array}{c}\text { Particle size }(\mu \mathrm{m}), \\
\text { Mean } \pm \text { S.D. }\end{array}$ & $\begin{array}{c}\text { \% ibuprofen released in } \\
\text { HCl solution in } 2 \mathrm{~h} \\
\text { Mean } \pm \text { S.D. }\end{array}$ & $\begin{array}{c}\mathrm{t}_{80 \%} \\
(\mathrm{~min})\end{array}$ & $\mathrm{k}$ & $\mathrm{n}$ & $\begin{array}{c}\text { Correlation } \\
\text { coefficient }\left(\mathrm{r}^{2}\right)\end{array}$ \\
\hline CALG & $1360 \pm 51.57$ & $11.43 \pm 2.09$ & 96 & 0.0154 & 0.7897 & 0.9977 \\
$0.5 \%$ EDA-CALG & $1273 \pm 75.18$ & $14.80 \pm 2.12$ & 150 & 0.0080 & 0.9229 & 0.9982 \\
$1.0 \%$ EDA-CALG & $1160 \pm 64.37$ & $17.85 \pm 1.98$ & 240 & 0.0071 & 0.9055 & 0.9999 \\
$2.0 \%$ EDA-CALG & $1,050 \pm 50.67$ & $18.37 \pm 2.01$ & 254 & 0.0057 & 0.9102 & 0.9954 \\
\hline
\end{tabular}

of ibuprofen in the microspheres was reduced. Such reduction in entrapment efficiency is probably due to higher alkalinity of the curing fluid $(\mathrm{pH} 9.0$ - 9.5) used for the preparation of EDA-CALG microspheres. Probably, the weakly acidic drug, ibuprofen leached out of the EDA-CALG microspheres to the external gelation medium at a much faster rate during preparation and lead to less drug entrapment efficiency (Table 1). Because, the $\mathrm{pH}$ of aqueous EDA solution was higher compared to mixtures of EDA-calcium chloride, separate solutions of EDA were not considered for the crosslinking of alginate matrix.

The release profile of CALG and EDA-CALG microspheres in $\mathrm{pH} 6.8$ phosphate buffers has been shown in Fig. 4 . The time point approach $\left(\mathrm{t}_{80 \%}\right.$ i.e. time required for the release of $80 \%$ drug) was used for the comparison of dissolution data. The drug release in phosphate buffer was faster in CALG microspheres than EDA-CALG microspheres as experienced from their $t_{80 \%}$ values (Table 2). The microspheres prepared with highest concentration of EDA exhibited the slowest drug release profile.

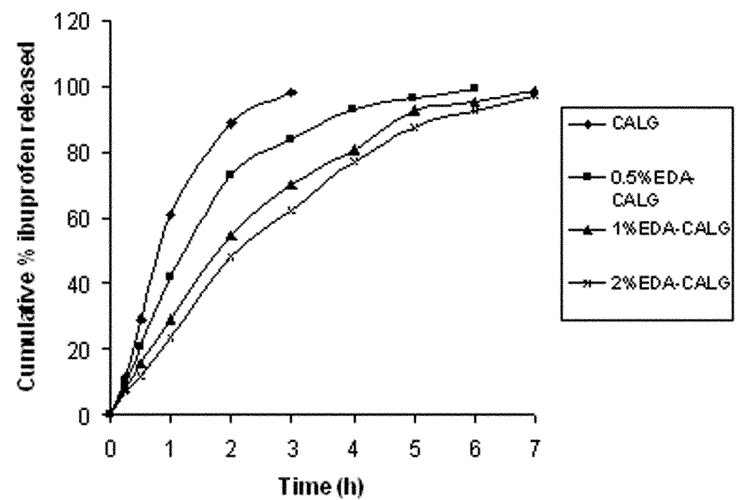

Fig. 4. Release profiles of ibuprofen-loaded CALG and EDA-CALG microspheres in phosphate buffer (pH 6.8).

The more sustained release of ibuprofen from EDA-reinforced CALG microspheres probably reflect the strengthening of the microspheres by ionic interaction of EDA $\left(\mathrm{NH}_{3}{ }^{+}\right)$with alginate (COO) ions. A similar explanation has been given for the sustained release of ampicillin from chitosan-treated alginate beads (Anal and Stevens, 2005). However, all formulations were capable of retarding the drug release below $18.37 \%$ for $2 \mathrm{~h}$ in $\mathrm{pH} 1.2 \mathrm{HCl}$ solutions 
(Table 2). The drug release from CALG microspheres was found lower in acidic media because of the limited solubility of calcium alginate in acidic media (Yotsuyanagi et al., 1987) and weakly acidic nature of the drug. The data obtained from in vitro release study in phosphate buffer ( $\mathrm{pH}$ 6.8) was fitted into Koresmeyer-Peppas equation to evaluate the release kinetics. It was found that the values of release exponent ' $n$ ' for the release of ibuprofen from both CALG and EDA-CALG microspheres range from 0.7897 to 0.9229 (Table 2). This indicated that the drug release from these systems follows anomalous transport through swelling and erosion of the polymer matrix. The values of release rate constant ' $\mathrm{k}$ ' for EDA-CALG microspheres decreased due to higher crosslinking of the polymer network compared to CALG microspheres. The physical state of the drug was evaluated through usual spectroscopic techniques such as FT-IR and DSC. The FT-IR spectrum (Fig. 5) showed the characteristic band of carbonyl $(\mathrm{C}=\mathrm{O})$ stretching band of isopropionic acid group of ibuprofen at $1719 \mathrm{~cm}^{-1}$
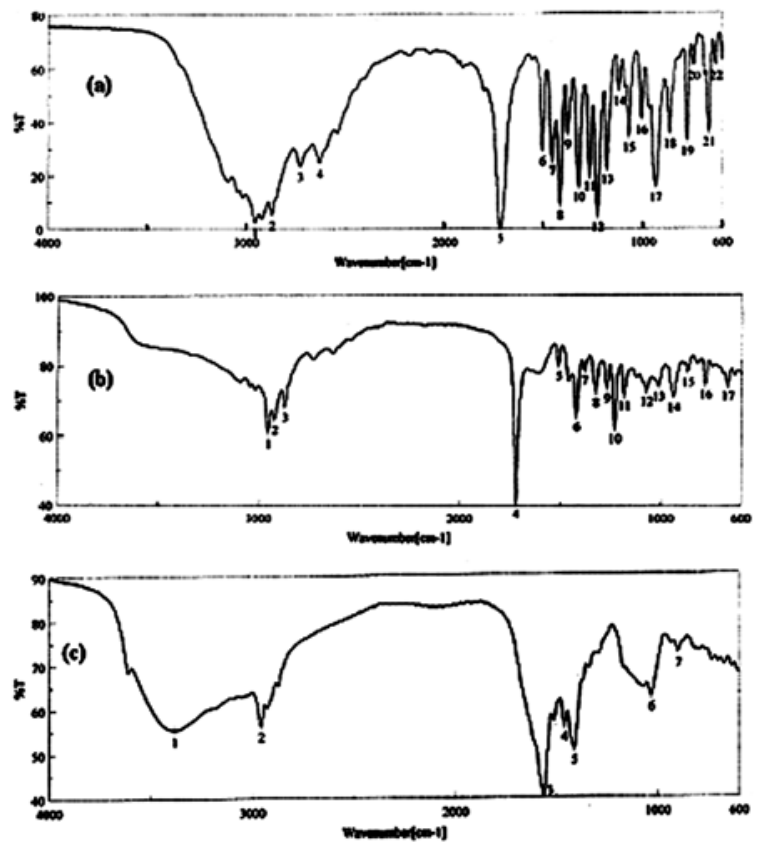

Fig. 5. FT-IR spectrum for (a) pure ibuprofen (b) ibuprofen-loaded CALG microspheres (c) ibuprofenloaded EDA-CALG microspheres. in pure ibuprofen (Pignatello et al., 2004) as well as ibuprofen-loaded CALG microspheres. The absorption of O-H stretching appeared also as a broad band near 2,500-3,000 $\mathrm{cm}^{-1}$. However, the characteristic band of $\mathrm{C}=\mathrm{O}$ stretching vibration of isopropionic acid group was disappeared from the spectrum of EDA-CALG microspheres. This fact could be attributed to greater ionization of carboxyl group of ibuprofen in EDA induced alkaline media. This fact was further confirmed by the appearance of band near $1562 \mathrm{~cm}^{-1}$ which is a characteristic band of $\mathrm{C}=\mathrm{O}$ stretching vibration of carboxylate anion $\left(\mathrm{COO}^{-}\right)$. An acid-base reaction between the carboxylic acid-containing drugs and neusilin has been indicated where FT-IR data showed a reduction in the absorbance of the free and the hydrogenbonded acid carbonyl peaks (Gupta et al., 2003). DSC thermograms of pure ibuprofen, ibuprofenloaded CALG and EDA-CALG microspheres have been represented in Fig. 6. The pure ibuprofen showed the melting endothermic peak at $78.09^{\circ} \mathrm{C}$ which was close to the reported melting point range of ibuprofen $\left(75-77^{\circ} \mathrm{C}\right)$. However, the CALG and EDA-CALG microspheres showed weaker melting endotherms of ibuprofen at $76.78^{\circ} \mathrm{C}$ and $79.69^{\circ} \mathrm{C}$, respectively. Thus, the thermal behavior of the microspheres studied within the temperature range $\left(30-140^{\circ} \mathrm{C}\right)$ helps to suggest that both the EDA-treated and untreated polymer might have

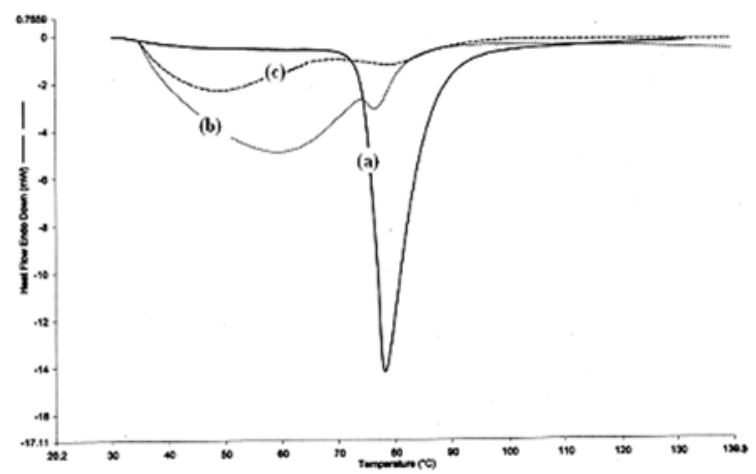

Fig. 6. DSC thermograms for (a) pure ibuprofen; (b) ibuprofen-loaded CALG microspheres; (c) ibuprofenloaded 2\% EDA-CALG microspheres. 
inhibited the melting of ibuprofen crystals close to its reported melting range. Such a thermal behavior may also be attributed to the reduction in the degree of ibuprofen crystallinity in the microspheres, indicating the presence of both crystalline and amorphous form of drug. Similar type of observations has been reported for the ibuprofen-loaded ethylcellulose/polystyrene microspheres (Saravanan et al., 2003).

\section{CONCLUSION}

A simple and mild ionotropic gelation method can be used for the preparation of ibuprofen-loaded CALG and EDA-CALG microspheres. This study indicated that the crosslinker EDA whose backbone contains two positively charged tertiary amino groups can interact with drugs having anionic functionalities (COO) and the degree of crystallinity may be reduced in the microspheres. Although, EDA sustained the release of ibuprofen for $7 \mathrm{~h}$ at $1 \%$ and $2 \% \mathrm{v} / \mathrm{v}$ concentration, drug entrapment efficiency was found lower. The release of ibuprofen followed non-Fickian kinetics even after EDA treatment. Further study is required to increase the drug entrapment efficiency and modify the drug release from EDA-CALG microspheres.

\section{ACKNOWLEDGMENTS}

The authors are grateful to the University Grant Commission for providing financial help for this work. The authority of Gupta College of Technological Sciences, Asansol, India and Jadavpur University, Department of Pharmaceutical Technology, Kolkata, India provided all facilities necessary for the work.

\section{REFERENCES}

Acarturk F, Takka S. (1999) Calcium alginate microparticles for oral administration: II. Effect of formulation factors on drug release and drug entrapment efficiency. J. Microencapsul. 16, 291-301.
Anal AK, Bhopatkar D, Tokura S, Tamura H, Stevens WF. (2003) Chitosan-alginate multilayer beads for gastric passage and controlled intestinal release of protein. Drug Dev. Ind. Pharm. 29, 713-724.

Anal AK, Stevens WF. (2005) Chitosan-alginate multilayer beads for controlled release of ampicillin. Int. J. Pharm. 290, 45-54.

Arica B, Calis S, Atilla P, Durlu NT, Cakar N, Kas HS, Hincal AA. (2005) In vitro and in vivo studies of ibuprofen-loaded biodegradable alginate beads. J. Microencapsul. 22, 153-165.

Bhopatkar D, Anal AK, Stevens WF. (2005) Ionotropic alginate beads for controlled intestinal protein delivery: Effect of chitosan and barium counterions on entrapment and release. J. Microencapsul. 22, 91-100.

Bodmeier R, Wang J. (1993) Microencapsulation of drugs with aqueous polymer dispersions. J. Pharm. Sci. 82, 191-194.

Calis S, Arica B, Kas HS, Hincal AA. (2002) 5-flurouracil loaded alginate microspheres in chitosan gel for local therapy of breast cancer. In: Chitosan in pharmacy and chemistry, edited by Muzzarelli RAA, Muzzarelli C, pp. 65-69, Atec, Italy.

Cary R, Dobson S, Delic J. (1999) 1, 2-diaminoethane (Ethylenediamine), International Programme on Chemical Safety (IPCS): Concise International Chemical Assessment Document; 15, World health organization, Geneva.

Coppi G, Lannuccelli V, Leo E, Bernabei MT, Cameroni R. (2001) Chitosan-alginate microparticles as a protein carrier. Drug Dev. Ind. Pharm. 27, 393400.

Davis SS, Hardy JG, Taylor MJ, Whalley DR, Wilson CG. (1984) A comparative study of the gastrointestinal transit of a pellet and tablet formulation. Int. J. Pharm. 21, 167-177.

Dubernet C, Benoit JP, Peppas NA, Puisieux F. (1995) Ibuprofen loaded ethylcellulose/polystyrene microspheres: An approach to get prolonged drug release excipients loaded alginate beads. Int. J. Pharm. 116, 125-128.

El-Gibaly I, Anwar MM. (1998) Development, characterization and in vivo evaluation of polyelectrolyte complex membrane gel microcapsules containing melatonin-resin complex for oral use. Bull. Pharm. Sci. 21, 117-139. 
Follonier N, Doelkar E. (1992) Biopharmaceutical comparison of oral multiple unit and single unit sustained release dosage forms. STP Pharm. Sci. 2, 141-158.

Gombotz WR, Wee SF. (1998) Protein release from alginate matrices. Adv. Drug Deliv. Rev. 31, 267-285.

Gupta MK, Vanwert A, Bogner RH. (2003) Formation of physically stable amorphous drugs by milling with neusilin. J. Pharm. Sci. 92, 536-551.

Gursoy A, Cevik S. (2000) Sustained release properties of alginate microspheres and tabletted microspheres of diclofenac sodium. J. Microencapsul. 17, 565-575.

Halder A, Mukherjee S, Sa B. (2005) Development and evaluation of polyethyleneimine-treated calcium alginate beads for sustained release of diltiazem. J. Microencapsul. 22, 67-80.

Kikuchi A, Kawabuchi M, Sugihara M, Sakurai Y. (1997) Pulsed dextran release from calcium-alginate gel beads. J. Control Release 47, 21-29.

Korsmeyer RW, Gurny R, Doelker EM, Buri P, Peppas NA. (1983) Mechanism of solute release from porous hydrophilic polymers. Int. J. Pharm. 15, 25-35.

Ostberg T, Lund EM, Graffner C. (1994) Calcium alginate matrices for multiple unit administration: IV. Release characteristics in different media. Int. J. Pharm. 112, 241-248.

Padmanabhan K, Smith TJ. (2002) Preliminary investigation of modified alginates as a matrix for gene transfection in a HeLa cell model. Pharm. Dev. Technol. 7, 97-101.

Peppas NA. (1985) Analysis of Fickian and nonFickian drug release from polymers. Pharm Acta Helv 60, 110-111.

Pignatello R, Spadaro D, Vandelli MA, Forni F, Puglisi. (2004) Characterization of the mechanism of interaction in ibuprofen-eudragit RL100 ${ }^{\circledR}$ coevaporates. Drug Dev. Ind. Pharm. 30, 277-288.

Rajaonarivony M, Vauthier C, Couarraze G, Puisieux F, Couvreur. (1993) Development of a new drug carrier made from alginate. J. Pharm. Sci. 82, 912-916.

Robinson D, De S. (2003) Polymer relationship during preparation of chitosan-alginat and poly- 1-lysinealginate nanospheres. J. Control Release 89, 101-112.

Ronan JM, Thompson SA. (2000) Medical devices comprising ionically and non-ionically crosslinked polymer hydrogels having improved mechanical properties. United States Patent, 6060534.
Sa B, Mondal UK, Prasad NR, Jha T. (1996) Development of indomethacin and ibuprofen loaded polymethyl methacrylate microparticles. Pharm. Sci. 2, 209-213.

Sa B. (1991) Studies on the release of theophylline from polyvinyl acetate microspheres. Drug Dev. Ind. Pharm. 27, 893-900.

Saravanan M, Bhaskar K, Srinivasa Rao, Dhanaraju MD. (2003) Ibuprofen-loaded ethylcellulose/polystyrene microspheres: An approach to get prolonged drug release with reduced burst effect and low ethylcellulose content. J. Microencapsul. 20, 289-302.

Sheftel VO. (1995) Handbook of Toxic Properties of Monomers and Additives. New York: CRC Press/ Lewis Publishers, p. 210.

Sinha VR, Trehan A. (2003) Biodegradable microspheres for protein delivery. J. Control Release 90, 261-280.

Takka S, Acarturk F. (1999) Calcium alginate microparticles for oral administration: I: Effect of sodium alginate type on drug release and drug entrapment efficiency. J. Microencapsul. 16, 275-290.

Tamilvanan S, Sa B. (1999) Effect of production variables on the physical characteristics of ibuprofenloaded polystyrene microparticles. J. Microencapsul. 16, 411-418.

Tanihara M, Suzuki Y, Yamamoto E, Noguchi A, Mizushima Y. (2001) Sustained release of basic fibroblast growth factor and angiogenesis in a novel covalently crosslinked gel of heparin and alginate. J. Biomed. Mater. Res. 56, 216-221.

Thu B, Bruheim P, Espevik T, Smidsrod O. SoonShiong P, Skjak-braek G. (1996) Alginate polycation microcapsules. II. Some functional properties. Biomaterials 17, 1069-1079.

Weib G, Knoch A, Laicher A, Stanislans F, Daniels R. (1993) Influence of polymer charge density on the simple coacervation of cellulose acetate phthalate. Eur. J. Pharm. Biopharm. 39, 239-243.

Yang RSH, Garman RH, Maronpot RR, McKelvey JA, Weil CS, Woodside MD. (1983) Acute and subchronic toxicity of ethylenediamine in laboratory animals. Fundam. Appl. Toxicol. 3, 512-520.

Yotsuyanagi T, Ohkubo T, Ohhashi T, Ikeda K. (1987) Calcium induced gelation of alginic acid and $\mathrm{pH}$ sensitive reswelling of dried gels. Chem. Pharm. Bull. 35, 1555-1563. 